\title{
Investigation on the Binding and Conformational Change of All-trans-Retinoic Acid with Peptidyl Prolyl cis/trans Isomerase Pin1 Using Spectroscopic and Computational Techniques
}

\author{
GuoFei Zhu, ${ }^{1}$ ShaoLi Lyu, ${ }^{2}$ Yang Liu, ${ }^{3}$ Chao Ma, ${ }^{1}$ and Wang Wang $\mathbb{D}^{3}$ \\ ${ }^{1}$ Institute of Food and Drug Manufacturing Engineering, Guizhou Institute of Technology, Guiyang 550025, China \\ ${ }^{2}$ Department of Ecology and Resource Engineering, Hetao College, Bayannur, Inner Mongolia 015000, China \\ ${ }^{3}$ Key Laboratory of Bio-resources and Eco-environment of the Ministry of Education, College of Life Sciences, Sichuan University, \\ Chengdu 610064, China
}

Correspondence should be addressed to Wang Wang; wangwang.jeff@hotmail.com

Received 26 June 2021; Revised 8 September 2021; Accepted 23 November 2021; Published 16 December 2021

Academic Editor: Vincenza Crupi

Copyright (C) 2021 GuoFei Zhu et al. This is an open access article distributed under the Creative Commons Attribution License, which permits unrestricted use, distribution, and reproduction in any medium, provided the original work is properly cited.

\begin{abstract}
Binding and conformational change of all-trans-retinoic acid (ATRA) with peptidyl prolyl cis/trans isomerase Pin1 were investigated systematically by spectroscopic and computational techniques under experimentally optimized physiological conditions. The intrinsic fluorescence of Pin 1 was quenched through a static quenching mechanism in the presence of ATRA with binding constants on the order of $10^{5} \mathrm{~mol} / \mathrm{L}$. Thermodynamic parameters $(\Delta H=15.76 \mathrm{~kJ} / \mathrm{mol}$ and $\Delta S=158.36 \mathrm{~J} / \mathrm{mol} \cdot \mathrm{K}$ at $293 \mathrm{~K})$ and computational results illustrated that the hydrophobic interactions played a significant role in the binding process of ATRA to Pin1, but electrostatic forces, weak van der Waals, and hydrogen bonds cannot be ignored. Circular dichroism, fluorescence spectra, and computational simulations revealed that ATRA interacted with residues Lys63 and Arg69 of Pin 1 to affect its conformational changes. Molecular dynamic simulation, principal component analysis, and free energy landscape monitored the dynamical conformational characteristics of ATRA binding to Pin1. All in all, the present research might provide a reference for the development and design of retinoic acid drugs that inhibit the activity of Pin1.
\end{abstract}

\section{Introduction}

Peptidyl prolyl cis-trans isomerase Pin1 is a unique enzyme that catalyzes cis-trans isomerization of phosphorylated serine/threonine-proline (pSer/Thr-Pro) motif and posttranslationally modulates the structure and function of Pin 1 substrates [1]. For example, Pin1 binds to pThr286-Pro motif of Cyclin D1 and increases its stability in the nucleus [2]. Moreover, Pin 1 binds to pSer246-Pro motif of $\beta$-catenin, inhibits its interaction with adenomatous polyposis coli (APC), and improves its stability and transport to the nucleus [2]. Therefore, Pin1 can regulate multiple cancerdriving signaling pathways, such as Wnt/ $\beta$-catenin, PI3K/AKT, and RTK/Ras/ERK pathway, and some physiological processes, such as cell cycle, apoptosis, and aging [3-5]. Pin1 contains 163 amino acid residues, which are composed of the WW domain and PPIase domain
(Figure 1(a)) [6]. The WW domain recognizes the pSer/ThrPro motif of the substrate and passes it to the PPIase domain for the catalytic substrate $[7,8]$. As a regulatory factor, Pin1 plays an important role in malignant tumors and neurodegenerative diseases, so it is an attractive and valuable drug target [9-11].

ATRA, one of the most active metabolites of vitamin A, has broad application prospects in cancer therapy and prevention [12]. ATRA belongs to the retinoid family, which is composed of a $\beta$-ionone ring and a polyunsaturated side with a carboxylic acid group (Figure 1(b)) [13]. A lot of literature has been reported the chemotherapeutic and chemopreventive effects of ATRA in hepatocellular carcinoma [14], breast cancer [15], gastric cancer [16], colon cancer [17], and prostate cancer [18]. Indeed, ATRA has become the standard front-line drug used for the treatment of acute promyelocytic leukemia (APL) in adults and that of 


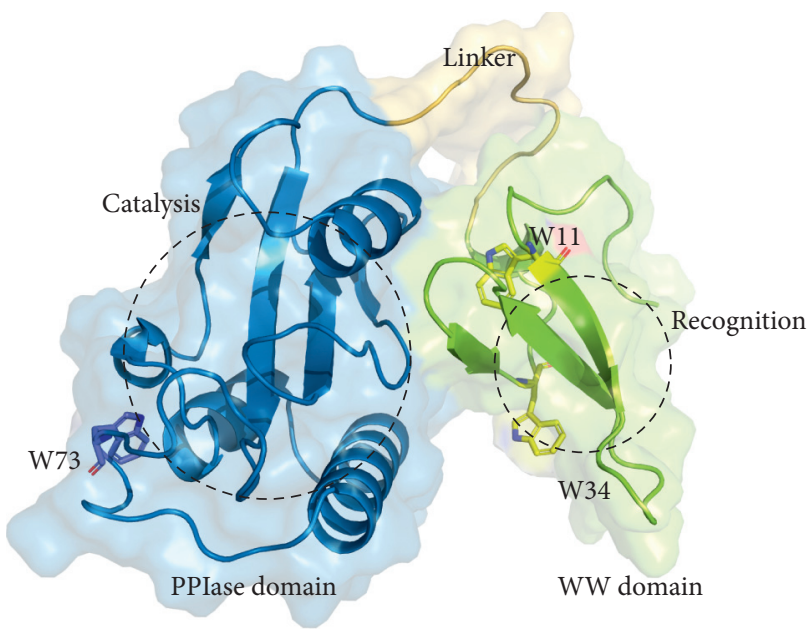

(a)<smiles>CC1=C(/C=C/C(C)=C/C=C/C(C)=C/C(=O)O)C(C)(C)CCC1</smiles>

(b)

FIgURE 1: The crystal structure of Pin1 (a) and chemical structure of ATRA (b). W11, W34, and W73 are three tryptophan residues.

Neuroblastoma in children [13]. Although ATRA exhibits a wide range of active functions, its application in other tumors is severely limited due to side effects, short half-life, and poor water solubility $[12,19]$.

Several researchers have illustrated that ATRA is one of the most potent Pin1 inhibitors to cure cancer [12-14]. Notably, ATRA binds to the active pocket of Pin 1 and inhibits its biological function, which induces its degradation in acute promyelocytic leukemia (APL) cells [20]. The combination of arsenic trioxide (ATO) and ATRA has also been reported to safely treat APL by targeting Pin1 [21]. The crystal structure of Pin1-ATRA complex reveals that ATRA binds to the catalytic domain of Pin1, but its specific inhibitory mechanism, thermodynamic parameters, binding affinity, energy transfer, and conformational changes are still unclear [22].

In the present work, we utilized multiple spectroscopic and computational techniques to explore the dynamical conformational characteristics of ATRA binding to Pin 1 in an aqueous solution at physiological conditions. The quenching constants $\left(K_{s v}\right)$, binding constants $\left(K_{a}\right)$, the number of binding sites $(n)$, and thermodynamic parameters $(\Delta H, \Delta S$, and $\Delta G)$ of Pin 1 by ATRA were calculated using fluorescence spectra at different temperatures (293 K and $303 \mathrm{~K})$. The conformational changes of ATRA binding to Pin 1 were determined by synchronous fluorescence, threedimensional (3D) fluorescence, and circular dichroism (CD). The dynamic characteristics of ATRA binding to Pin1 were monitored at the atomic level by molecular dynamics simulations, principal component analysis, and free energy landscape. This study would help understand the binding model and inhibition mechanism of Pin1-ATRA complex.

\section{Materials and Methods}

2.1. Materials. The standard of ATRA was purchased from Macklin Biochemical Co., Ltd. (Shanghai, China). Yeast extract and peptone were products of AoBox Biotechnology Co., Ltd. (Beijing, China). His-tag purification resin and ultrafiltration spin columns were gained from Beyotime Biotechnology Co., Ltd. (Shanghai, China). Thermolysin was obtained from Yuanye Biotechnology Co., Ltd. (Shanghai, China). Isopropyl-beta-D-thiogalactopyranoside (IPTG), penicillin, and other reagents were obtained from Solarbio Technology Co., Ltd. (Beijing, China).

2.2. Preparation of Pin1. The expression and purification of Pin 1 were shown as described before $[23,24]$. Briefly, wild type Pin1 (WT-Pin1) and ten alanine mutants (H59A, L61A, K63A, R68A, R69A, C113A, Q131, M130A, F134A, and H157A) were expressed in E. coli BL21 (DE3), which contains the recombinant plasmid pET-19b-Pin1. Then, interesting proteins were purified using His-tag purification resin and ultrafiltration spin columns, which were placed in Buffer C (25 mM Tris, $200 \mathrm{mM} \mathrm{NaCl}, \mathrm{pH}$ 7.4). Meanwhile, the purities of Pin 1 and mutants were detected using SDS-PAGE (purity $>90 \%$ ). The concentrations of Pin 1 and mutants were measured using Bradford assay.

2.3. Spectral Measures. Circular dichroism (CD) measurements were carried out on Jasco J-815 circular dichroism spectropolarimeter (JASCO, Japan) with a quartz cell of $0.1 \mathrm{~cm}$ path length at $298 \mathrm{~K}$. The spectra range, scan speed, and bandwidth were set to $200-250 \mathrm{~nm}, 20 \mathrm{~nm} / \mathrm{min}, 1.0 \mathrm{~nm}$, respectively. The concentration of Pin1 ( $\mathrm{pH} 7.4$ ) was controlled at $10 \mu \mathrm{M}$; then ATRA was gradually added. Every spectrum was the mean of three spectra.

The fluorescence measurements were collected on F-4500 fluorescence spectrophotometer (Hitachi, Japan) with a $1.0 \mathrm{~cm}$ quartz cell and a thermostat bath. The concentration of Pin1 ( $\mathrm{pH} 7.4$ ) was controlled at $5 \mu \mathrm{M}$; then ATRA was gradually added. Fluorescence emission spectra were measured in the wavelength range of 310-400 $\mathrm{nm}$ when excitation wavelength $\left(\lambda_{\text {ex }}\right)$ was $295 \mathrm{~nm}$ at $293 \mathrm{~K}$ and $303 \mathrm{~K}$. In addition, synchronous fluorescence spectra were recorded in the wavelength range of $270-310 \mathrm{~nm}$ and $250-310 \mathrm{~nm}$ when $\Delta \lambda\left(\Delta \lambda=\lambda_{\mathrm{em}}-\lambda_{\mathrm{ex}}\right)$ was 15 and $60 \mathrm{~nm}$ at $293 \mathrm{~K}$, 
respectively. Also, the three-dimensional (3D) fluorescence spectra were recorded in the emission wavelength range of $200-450 \mathrm{~nm}$ and the excitation wavelength range of $200-350 \mathrm{~nm}$ at $293 \mathrm{~K}$. The excitation slit, emission slit, scanning speed, and voltage were set to $5 \mathrm{~nm}, 5 \mathrm{~nm}$, $1200 \mathrm{~nm} / \mathrm{min}$, and $700 \mathrm{~V}$, respectively. Every spectrum was the average of three spectra.

2.4. Computational Simulations. Classical molecular dynamic (MD) simulation was performed to study the binding and conformational changes of ATRA with peptidyl prolyl cis/trans isomerase Pin1. The crystal structure of Pin1-ATRA complex was downloaded from Protein Data Bank website (PDB_ID: 4TNS) [22]. PyMol was adopted to remove the water molecules and ions [25]. To construct the wild type structure, the residues Q77 and Q82 were replaced by residues K77 and K82 using Swiss-Model server [26]. MD simulations of Pin1 and Pin1-ATRA complex were performed in GROMACS v4.6.5 with AMBER99SB all-atom force field [27]. The pdb2gmx and antechamber programs were implemented to produce the topology files of Pin 1 and ATRA, respectively. Pin1 and Pin1-ATRA complex were solvated in a dodecahedron periodic box using TIP3P molecules. To keep the charge-neutral environment, $\mathrm{Cl}^{-}$was added in Pin1 and Pin1-ATRA systems. Energy minimization with steepest descent algorithm was performed with 5000 steps to correct improper geometries and avoid steric clashes. Then, $100 \mathrm{ps}$ NVT and NPT ensembles were used to balance system at $300 \mathrm{~K}$ and $1 \mathrm{~atm}$. Finally, $50 \mathrm{~ns}$ MD simulations of Pin1 and Pin1-ATRA complex began to run $50 \mathrm{~ns}$ with 2 fs timestep at $310 \mathrm{~K}$. The g_rms, g_rmsf, g_gyrate, and $g \_h b o n d$ programs were used to analyze the backbone root mean square deviation (RMSD), root mean square fluctuation (RMSF), radius of gyration (Rg), and hydrogen bonds, respectively.

2.5. Binding Energy Calculations. The MM/PBSA (Molecular Mechanics/Poisson-Boltzmann Surface Area) approach was executed to predict the binding free energy between Pin1 and ATRA. The last $5 \mathrm{~ns}$ trajectory of MD simulation was extracted with 100 snapshots. The following formula was implemented to estimate binding free energy $\left(\Delta G_{b i n d}\right)$ [28]:

$$
\begin{aligned}
\Delta G_{\text {bind }} & =\Delta G_{\text {complex }}-\left(\Delta G_{\text {protein }}+\Delta G_{\text {ligand }}\right) \\
& =\Delta E_{M M}+\Delta G_{\text {sol }}-T \Delta S, \\
\Delta E_{M M} & =\Delta E_{\text {vdw }}+\Delta E_{\text {ele }}, \\
\Delta G_{\text {sol }} & =\Delta G_{\text {polar }}+\Delta G_{\text {nopolar }} .
\end{aligned}
$$

We have that $\Delta G_{\text {complex }}, \Delta G_{\text {protein, }}$, and $\Delta G_{\text {ligand }}$ are total free energy of Pin1-ATRA complex, Pin1, and ATRA, respectively. $\Delta E_{M M}$ consists of van der Waals $\left(\Delta E_{v d w}\right)$ and electrostatic interaction energy $\left(\Delta E_{\text {ele }}\right) . \Delta G_{\text {sol }}$ consists of polar solvation free energy $\left(\Delta \mathrm{G}_{\text {polar }}\right)$ and nonpolar solvation free energy $\left(\Delta \mathrm{G}_{\text {noploar }}\right) . \mathrm{T} \Delta S$ is entropic contribution, which is neglected due to its high computational cost and low prediction accuracy in MM/PBSA approach.
Therefore, the binding free energy $\left(\Delta G_{b i n d}\right)$ consists of van der Waals $\left(\Delta E_{v d w}\right)$, electrostatic interaction energy $\left(\Delta E_{\text {ele }}\right)$, polar solvation free energy $\left(\Delta \mathrm{G}_{\text {polar }}\right)$, and nonpolar solvation free energy $\left(\Delta \mathrm{G}_{\text {noploar }}\right)$, respectively. It is expressed follows:

$$
\Delta G_{\text {bind }}=\Delta E_{v d w}+\Delta E_{\text {ele }}+\Delta G_{\text {polar }}+\Delta G_{\text {nonpolar }} .
$$

The g_mmpbsa program and python script MmPbSaStat.py were used to predict the binding energy between Pin1 and ATRA [29].

2.6. Principal Component Analysis and Free Energy Landscape. Principal component analysis (PCA) was implemented to study the essential motions of biomacromolecules during MD simulations [30, 31]. The correlation matrix $\mathrm{C}$ was constructed using the coordinates of $\mathrm{C} \alpha$ atoms and diagonalized to generate the eigenvalues and their corresponding eigenvectors from the 50 ns MD trajectories. Covariance matrix $(C)$ of $\mathrm{C} \alpha$ atoms from each residue was calculated as in the following equation [32]:

$$
C_{i j}=\left[M_{i i}^{1 / 2}\left(x_{i}-\left(x_{i}\right)\right) M_{j j}^{1 / 2}\left(x_{j}-\left(x_{j}\right)\right)\right],
$$

where $C$ is a symmetric $3 n \times 3 n$ matrix, $n$ is the number of residues, and $M$ is a diagonal matrix. The $g_{-}$covar package was adopted to construct and diagonalize covariance matrices of $\mathrm{C} \alpha$ atoms. The $g$ anaeig package was performed to project the trajectory onto the eigenvector. The first eigenvector and second eigenvector were considered as principal component 1 (PC 1) and principal component 2 (PC 2), respectively.

Free energy landscape (FEL) is an effective technique to study the conformational changes related to different energy states [32, 33]. PC 1 and PC 2 were implemented to construct the two-dimensional representation of FEL. The minimum value of free energy indicates a stable conformation, and the energy barrier connecting the minimum value suggests a metastable state. Gibbs free energy $(G \alpha)$ was obtained as in the following equation [34]:

$$
G_{\alpha}=-k T \ln P_{(q \alpha)} P_{\max }(q),
$$

where $k, T, P_{(q \alpha)}$, and $P_{\max }(q)$ are Boltzmann constant, absolute temperature, the probability density function, and the prospects of the most probable state, respectively. Gibbs free energy was generated using g_sham package.

2.7. Drug Affinity Responsive Target Stability (DARTS) Assay. DARTS assay can effectively detect the stability of protein binding to molecules because protein is less susceptible to proteolysis when it is drug-bound than when it is drug-free [35]. ATRA $(0.2 \mathrm{mg} / \mathrm{ml})$ was added to Pin 1 and its mutants $(0.2 \mathrm{mg} / \mathrm{ml})$ and incubated at room temperature for $30 \mathrm{~min}$. Then, the mixed solutions were proteolyzed at room temperature for $30 \mathrm{~min}$ with thermolysin $(1: 1000)$. Next, mixed solutions were detected by SDS-PAGE. 


\section{Results and Discussion}

\subsection{Fluorescence Spectroscopic Studies}

3.1.1. Fluorescence Quenching Mechanism. Fluorescence spectroscopy is an extremely useful and accurate method to monitor the binding and conformational changes of ligands with protein. It is well known that the endogenous fluorescence of protein is contributed mainly by tryptophan (Trp) and tyrosine (Tyr) residues. Also, when the excitation wavelength $\left(\lambda_{e x}\right)$ was $295 \mathrm{~nm}$, the fluorescence spectra showed the endogenous fluorescence of tryptophan residues [36, 37]. A previous study had revealed that the activity of Pin 1 decreased at high temperature, so this experiment selected $293 \mathrm{~K}$ and $303 \mathrm{~K}$ to implement the fluorescence spectra [38]. As shown in Figures 2(a) and 2(b), the fluorescence intensities of Pin1 were decreased gradually with increasing concentration of ATRA. When the concentration of ATRA was $5 \mu \mathrm{M}$, the fluorescence quenching rates were $50.60 \%$ and $59.46 \%$, respectively, at $293 \mathrm{~K}$ and $303 \mathrm{~K}$. The phenomenon revealed that ATRA interacted with Pin 1 and quenched its intrinsic fluorescence. Meanwhile, the result further confirmed the previous scientific reports that ATRA was an effective Pin1 inhibitor [20]. In addition, it was obvious that the maximum wavelength $(\lambda \max )$ of fluorescence spectra showed a tiny red-shift from $338.2 \mathrm{~nm}$ to $342.4 \mathrm{~nm}$ and from $339.0 \mathrm{~nm}$ to $342.4 \mathrm{~nm}$ at $293 \mathrm{~K}$ and $303 \mathrm{~K}$, respectively, with the increasing of ATRA. It indicated that the binding of ATRA induced a certain conformational change of Pin1, including a decrease in the hydrophobicity around the microenvironment of tryptophan residues.

In general, there are three fluorescence quenching mechanisms: static quenching mode, dynamic quenching mode, and combination of both modes. The quenching mechanism between Pin 1 and ATRA was discriminated using the following Stern-Volmer equation [39, 40]:

$$
\frac{F_{0}}{F}=1+K_{s v}[Q]
$$

where $F_{0}$ and $F$ are the maximum fluorescence intensity of Pin1 in the absence and presence of ATRA, respectively. $Q$ is the concentration of ATRA. $K_{s v}$ is a quenching constant.

Figure 2(c) shows a well-linear relationship between Pin1 and ATRA, which suggests that the quenching mechanism of ATRA is either a static quenching or a dynamic quenching mechanism. Scientific literature illustrates that quenching constant $\left(K_{s v}\right)$ decreasing with increasing temperature is static quenching, and the opposite is dynamic quenching $[41,42]$. From Table 1 , the $K_{s v}$ values are 1.83 and $1.32 \times 10^{5} \mathrm{~mol} / \mathrm{L}$ at $293 \mathrm{~K}$ and $303 \mathrm{~K}$, respectively, suggesting that the quenching mode between Pin1 and ATRA is a typical static quenching mechanism.

\subsubsection{Binding Constants and Thermodynamic Parameters.} The affinity of the drug-receptor is one of the significant indicators for evaluating drug efficacy, and it is closely related to the binding constant $(K a)$. As a static quenching process, binding constant was calculated using the following equation $[43,44]$ :

$$
\log \left[\frac{\left(F_{0}-F\right)}{F}\right]=\log K_{a}+n \log [Q],
$$

where $K_{a}$ and $n$ are the binding constant and the number of binding sites, respectively.

As shown in Figure 2(d) and Table 1, the $n$ values are approximately equal to 1 at $293 \mathrm{~K}$ and $303 \mathrm{~K}$. The observation was consistent with the reported crystal structure, indicating that Pin1 bound only one ATRA [22]. Also, the $K_{a}$ values are 2.90 and $2.06 \times 10^{5} \mathrm{~mol} / \mathrm{L}$ at $293 \mathrm{~K}$ and $303 \mathrm{~K}$, respectively. Our result was similar to the previous study, suggesting that there was a high affinity between ATRA and Pin1 [22]. In addition, the $K_{a}$ values display downtrend with the increasing temperatures, in agreement with the $K_{s v}$ values, further illustrating that the quenching mechanism between Pin 1 and ATRA is a static quenching process.

Binding forces were involved in the binding process of Pin 1 with ATRA. It is well known that the binding forces are mainly van der Waals forces, hydrogen bonds, hydrophobic interactions, and electrostatic forces. These binding forces were distinguished through thermodynamic parameters obtained using the following equation [45]:

$$
\begin{aligned}
\ln \Delta\left(\frac{K_{2}}{K_{1}}\right) & =\Delta H\left(\frac{1 / T_{1}-1 / T_{2}}{R}\right), \\
\Delta G & =-R T \ln K_{a} \\
\Delta S & =-\frac{(\Delta G-\Delta H)}{T},
\end{aligned}
$$

where $\Delta H, \Delta G$, and $\Delta S$ are enthalpy change, binding free energy, and entropy change, respectively. $R$ and $T$ are gas constant and experimental temperature, respectively.

According to the viewpoint of Ross and Subramanian [46], if $\Delta H$ and $\Delta S>0$, hydrophobic interactions were binding forces; if $\Delta H<0$ and $\Delta S>0$, electrostatic forces were binding forces; if $\Delta H<0$ and $\Delta S<0$, hydrogen bonds and van der Waals forces were binding forces. From Table 1, the values of $\Delta H$ and $\Delta S$ are $15.76 \mathrm{~kJ} / \mathrm{mol}$ and $158.36 \mathrm{~J} / \mathrm{mol} \cdot \mathrm{K}$ in the binding process of ATRA to Pin 1 at $293 \mathrm{~K}$, respectively. The phenomenon suggested that hydrophobic interactions were the main binding force between ATRA and Pin1. In addition, the binding force between Pin1 and ATRA was analyzed in detail through the following computational simulations (Figures 3 and 4). It is observed that the values of $\Delta G$ are -30.64 and $-29.80 \mathrm{~kJ} / \mathrm{mol}$ at $293 \mathrm{~K}$ and $303 \mathrm{~K}$, respectively, indicating that the binding of ATRA to Pin 1 is a spontaneous process.

\subsection{Conformational Studies}

3.2.1. Circular Dichroism. The binding of ligands to receptors not only affected their thermal stability but also changed their binding conformation. Circular dichroism (CD) is one of the most widely used techniques to investigate the secondary structure changes of protein-ligand complex [47]. As shown in Figure 5(a), CD spectra of Pin 1 have been decreased with the increasing amount of ATRA. It implied 


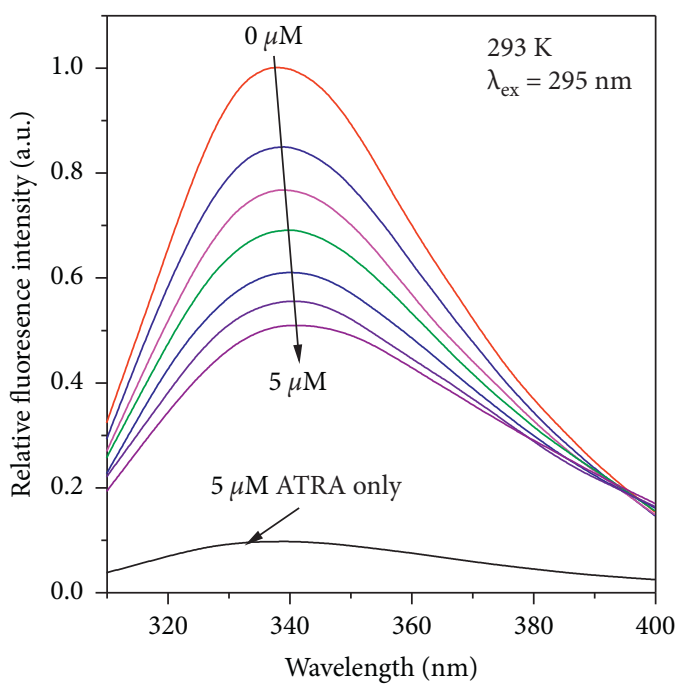

(a)

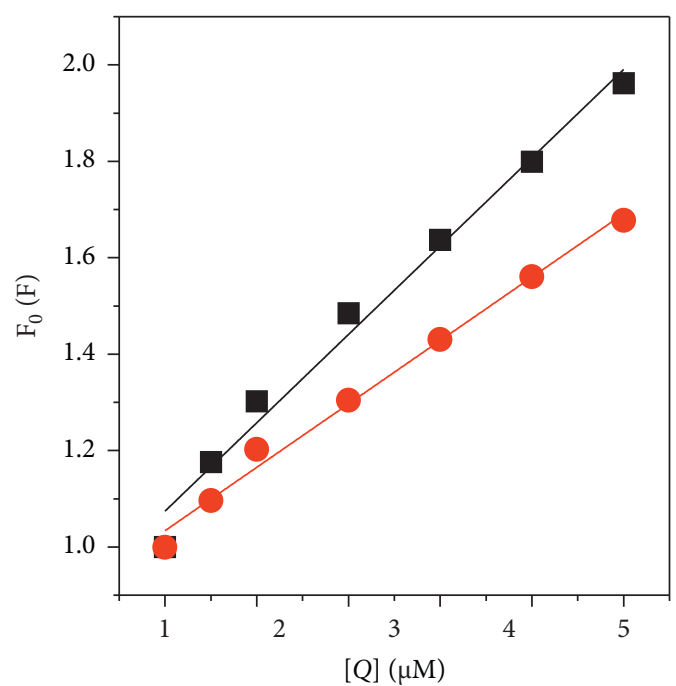

$293 \mathrm{~K}$

$303 \mathrm{~K}$

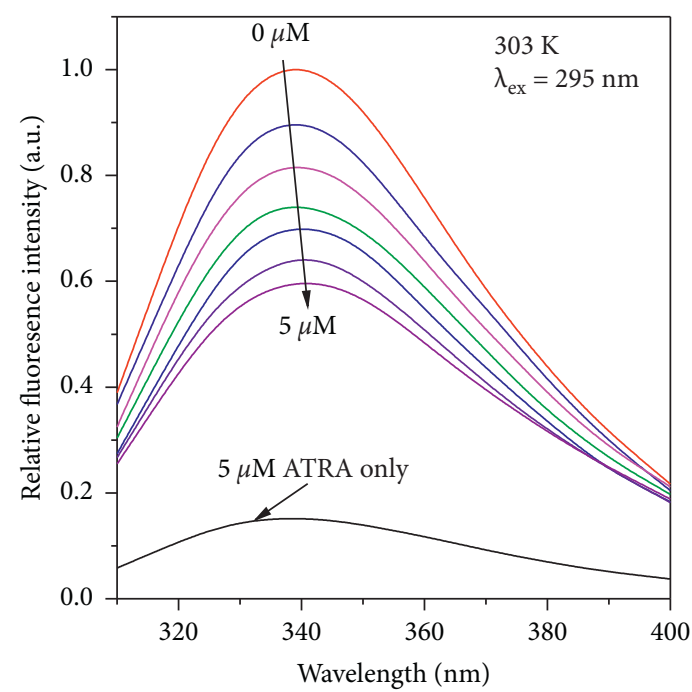

(b)

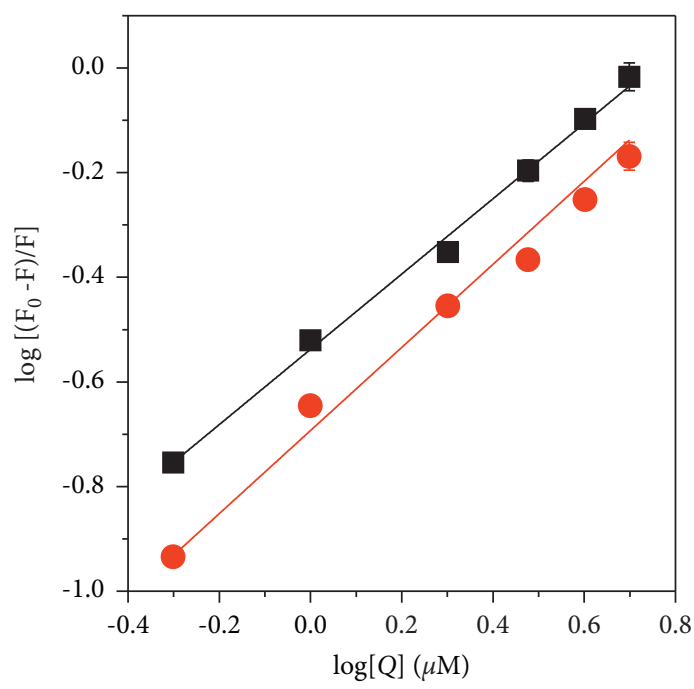

$293 \mathrm{~K}$

$303 \mathrm{~K}$

(c)

(d)

FIgURE 2: Fluorescence spectra of Pin1-ATRA complex. (a, b) Fluorescence emission spectra of Pin1 in the absence and presence of ATRA at $293 \mathrm{~K}$ and $303 \mathrm{~K}$, respectively. $\lambda_{e x}=295 \mathrm{~nm}, c_{\text {pin } 1}=5 \mu \mathrm{M}$, and $c_{\mathrm{ATRA}}=0$ (red line), 0.5 (blue line), 1 (magenta line), 2 (olive line), 3 (navy line), 4 (violet line), and 5 (purple line) $\mu \mathrm{M}$. (c) Stern-Volmer and (d) double logarithmic plot for the interaction of Pin1 and ATRA.

TABle 1: Thermodynamic parameters of the Pin1-ATRA complex at different temperatures.

\begin{tabular}{lcc}
\hline Parameters & $293 \mathrm{~K}$ & $303 \mathrm{~K}$ \\
\hline$K_{s v}\left(10^{5} \mathrm{~mol} / \mathrm{L}\right)$ & $1.83 \pm 0.01$ & $1.32 \pm 0.01$ \\
$R^{2 a}$ & 0.993 & 0.996 \\
$n$ & $0.73 \pm 0.02$ & $0.73 \pm 0.03$ \\
$K_{a}\left(10^{5} \mathrm{~mol} / \mathrm{L}\right)$ & $2.90 \pm 0.02$ & $2.06 \pm 0.01$ \\
$R^{2 b}$ & 0.998 & 0.996 \\
$\Delta G(\mathrm{~kJ} / \mathrm{mol})$ & $-30.64 \pm 0.09$ & $-29.80 \pm 0.34$ \\
$\Delta H(\mathrm{~kJ} / \mathrm{mol})$ & $15.76 \pm 0.88$ & $15.76 \pm 0.88$ \\
$\Delta S(\mathrm{~J} / \mathrm{mol} / \mathrm{K})$ & $158.36 \pm 0.34$ & $154.91 \pm 0.42$ \\
\hline
\end{tabular}




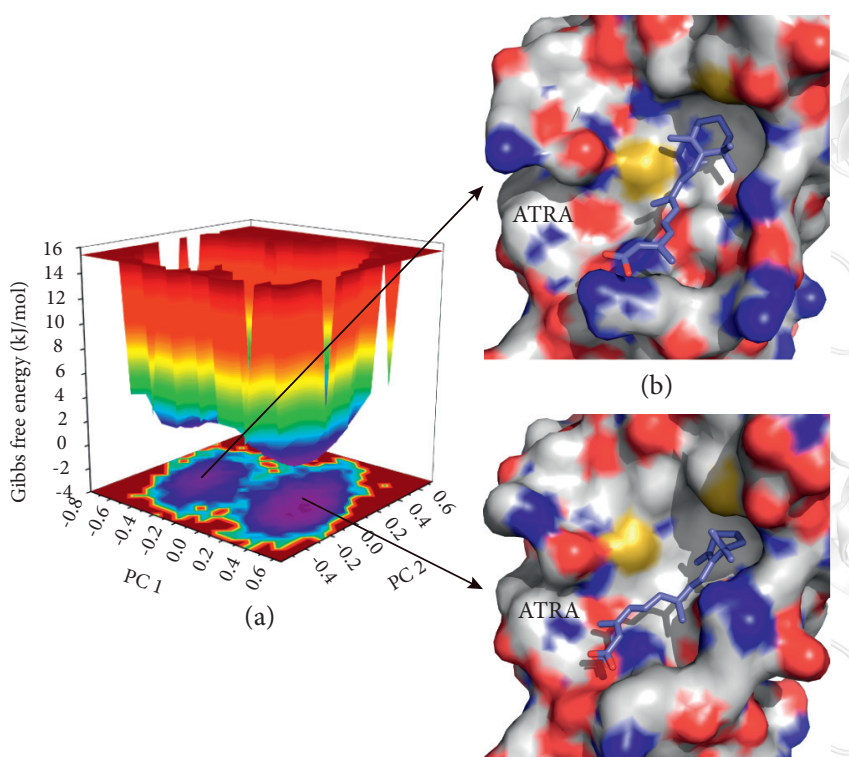

(e)

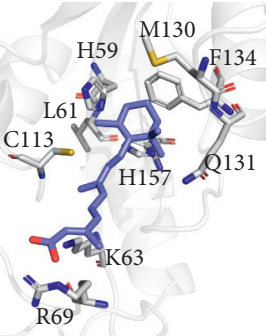

(c)

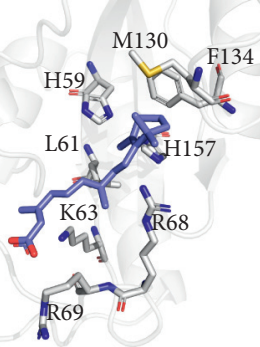

(f)

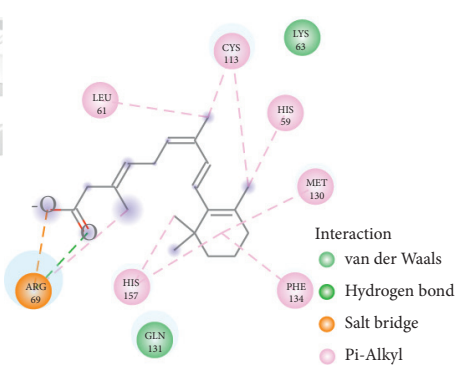

(d)

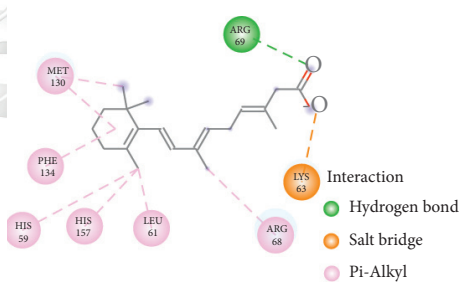

(g)

FIgure 3: Free energy landscape plot (a) and binding model (b)-(g) of Pin1-ATRA complex during MD simulation.

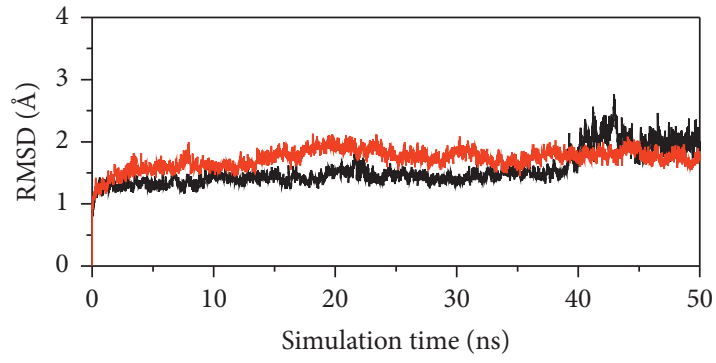

- Pin 1

— Pin1-ATRA

(a)

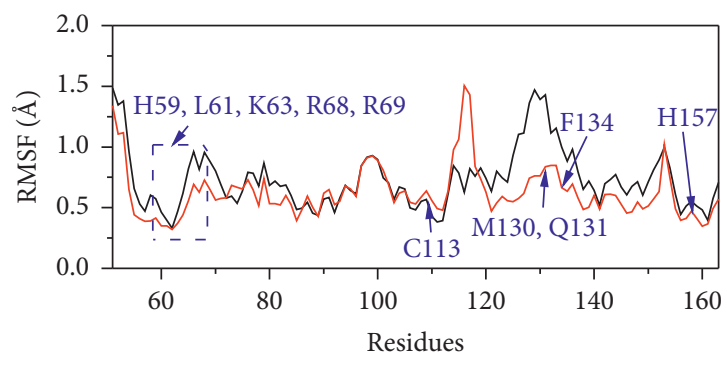

— Pin 1

- Pin1-ATRA

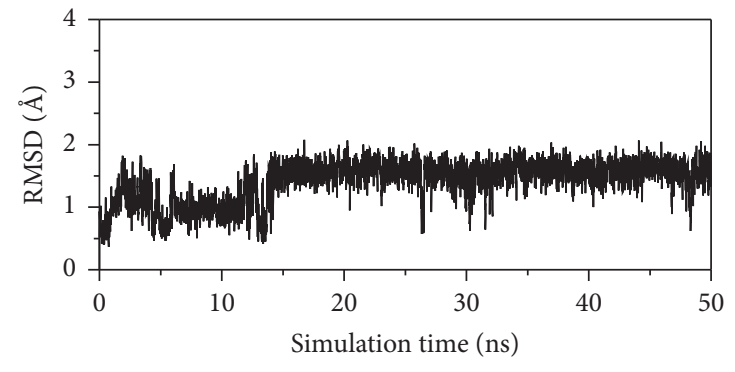

— ATRA

(b)

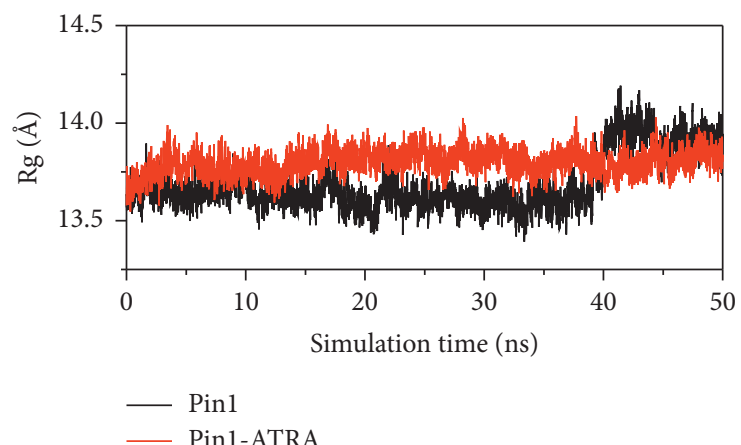

(d)

FIgURE 4: Continued. 


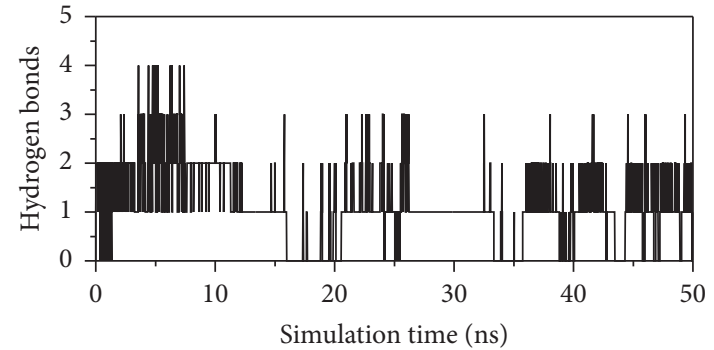

- Pin1-ATRA

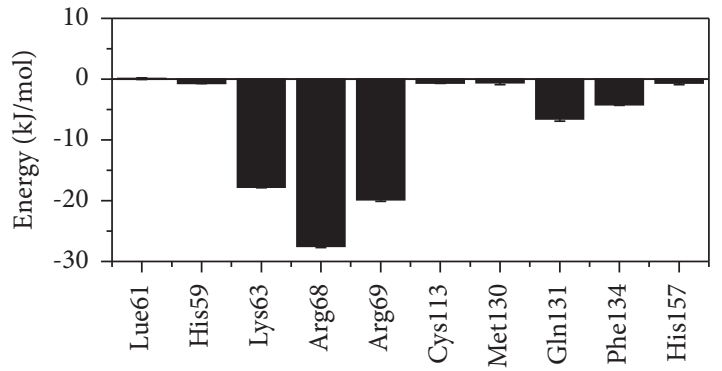

Pin1-ATRA

(e)

(f)

Figure 4: MD simulations of Pin1-ATRA complex. (a) RMSD of Pin1 and Pin1-ATRA complex during 50 ns MD simulation. (b) RMSD of ATRA during 50 ns MD simulation. (c) RMSF of Pin1 and Pin1-ATRA complex during last 5 ns MD simulation. (d) Rg of Pin1 and Pin1ATRA complex during 50 ns MD simulation. (e) Hydrogen bonds of Pin1-ATRA complex during 50 ns MD simulation. (f) Residues energy decomposition of Pin1-ATRA complex during 50 ns MD simulation.

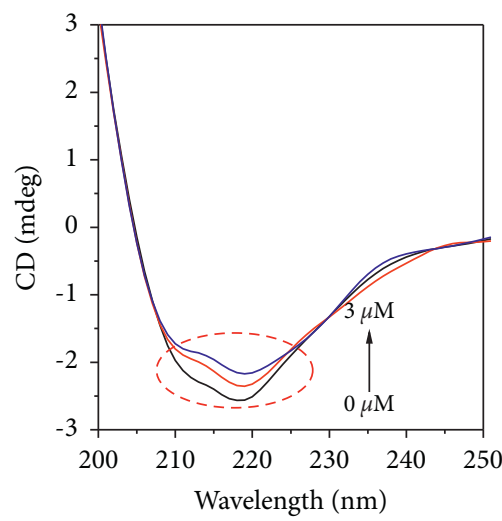

(a)

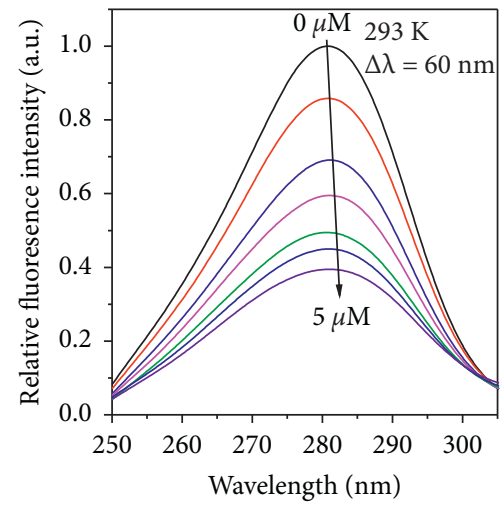

(c)

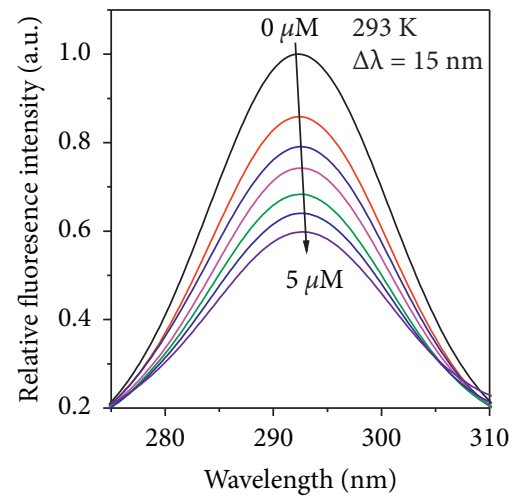

(b)

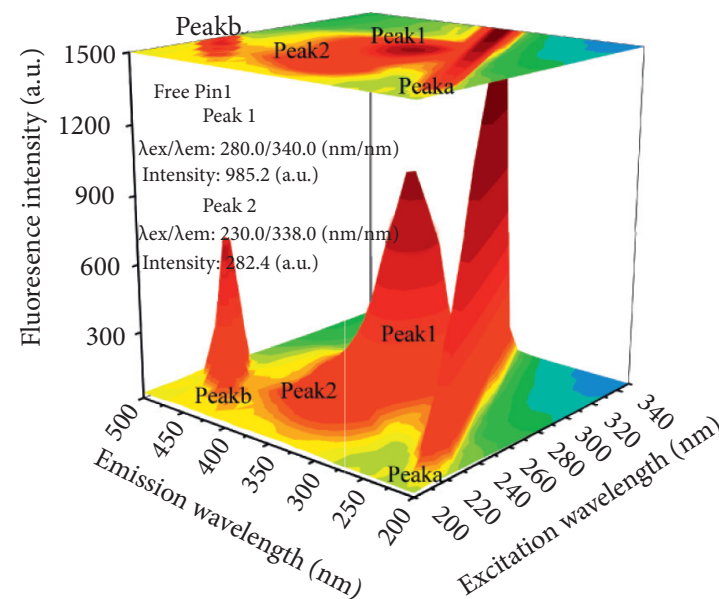

(d)

Figure 5: Continued. 


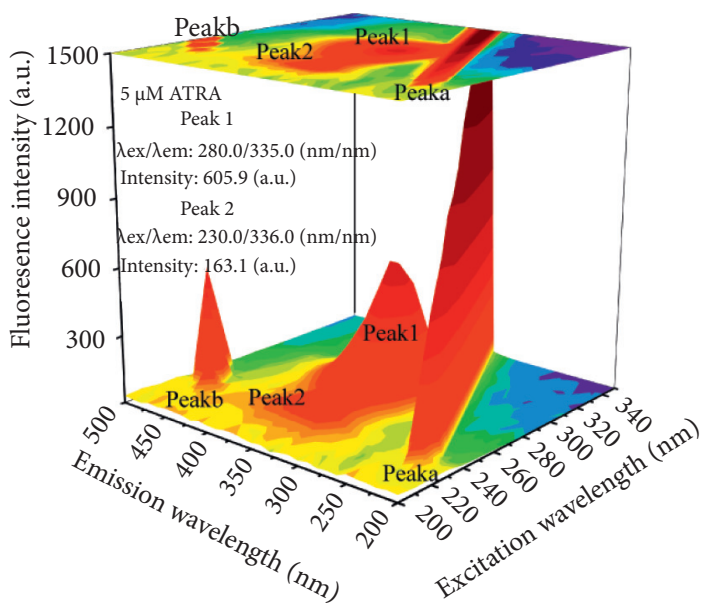

(e)

Figure 5: ATRA affects the conformation of Pin1. (a) CD spectra of Pin1 in the absence and presence of ATRA. $c_{\text {Pin } 1}=10 \mu M$; $c_{A T R A}=0$ (black line), 1 (red line), and 3 (blue line) $\mu \mathrm{M}$. (b, c) Synchronous fluorescence spectra of Pin 1 in the absence and presence of ATRA with $\Delta \lambda=15 \mathrm{~nm}$ and $60 \mathrm{~nm}$, respectively. $c_{\text {Pin } 1}=5 \mu \mathrm{M} ; c_{\text {ATRA }}=0$ (black line), 0.5 (red line), 1 (blue line), 2 (magenta line), 3 (olive line), 4 (navy line), and 5 (violet line) $\mu \mathrm{M}$. (d, e) Three-dimensional fluorescence spectra of Pin1 in the absence and presence of ATRA; $c_{\text {Pin } 1}=5 \mu \mathrm{M}$ and $\mathrm{c}_{\mathrm{ATRA}}=0$ and $5 \mu \mathrm{M}$.

that ATRA changed the secondary structure of Pin 1 and affected its stability. To explore the stability of the secondary structure, $\alpha$-helix contents of Pin1 were calculated using the following equation [41]:

$$
\begin{aligned}
\mathrm{MRE}_{208} & =\frac{\text { observed CD }(\text { mdeg })}{C_{p} \mathrm{nl} \times 10}, \\
\alpha-\operatorname{helix}(\%) & =\frac{-\mathrm{MRE}_{208}-4000}{33000-4000},
\end{aligned}
$$

where $\mathrm{MRE}_{208}$ is mean residue ellipticity (MRE) at $208 \mathrm{~nm}$ and $C_{p}, n$, and $l$ are the molar concentration of Pin 1 $(10 \mu \mathrm{mol} / \mathrm{L})$, the number of amino acid residues (163), and the path length of the cell $(0.1 \mathrm{~cm})$, respectively.

The calculated results showed that the $a$-helix content of Pin 1 declined from $23.33 \%$ to $19.89 \%$ with the increasing amount of ATRA. The helical stability of Pin 1 induced by ATRA might have altered hydrogen bonding networks, which resulted in unfolding of polypeptides and changing the secondary conformation of Pin1 [42].

\subsubsection{Synchronous Fluorescence Spectroscopy.} Synchronous fluorescence spectroscopy can detect the changes in the microenvironment of Pin 1 after interacting with ATRA [48]. Synchronous fluorescence spectroscopy provides mainly the conformational characteristics of Tyr and $\operatorname{Trp}$ residues when $\Delta \lambda=15$ and $60 \mathrm{~nm}$, respectively. As shown in Figures 5(b) and 5(c), the fluorescence intensities of Tyr and Trp residues were declined regularly with the increasing amount of ATRA. When the concentration of ATRA is $5 \mu \mathrm{M}$, the fluorescence quenching rates of Tyr and Trp residues are $59.78 \%$ and $39.47 \%$, respectively, at $293 \mathrm{~K}$. The results demonstrated that ATRA not only quenched the intrinsic fluorescence of Tyr residues but also decreased the intrinsic fluorescence of Trp residues.
It is noted that $\lambda_{\max }$ of fluorescence spectra of Tyr and Trp residues display a slight red-shift from $292.4 \mathrm{~nm}$ to $293.6 \mathrm{~nm}$ and from $280.8 \mathrm{~nm}$ to $282.0 \mathrm{~nm}$, respectively. The results, which were in accordance with endogenous fluorescence (Figures 2(a) and 2(b)), illustrated that the ATRA declined the hydrophobicity and increased the polarity around the Tyr and Trp residues in Pin1.

\subsubsection{Three-Dimensional Fluorescence Spectroscopy.} Three-dimensional (3D) fluorescence spectroscopy is widely implemented to explore the conformational changes of protein-ligand complex. The 3D fluorescence spectra and characteristic peaks are displayed in Figures 5(d) and 5(e). It can be seen that Pin 1 has four characteristic peaks in the absence and presence of ATRA. Peak a and peak b represent the first-order Rayleigh scattering peak $\left(\lambda_{\mathrm{ex}}=\lambda_{\mathrm{em}}\right)$ and the second-order Rayleigh scattering peak $\left(2 \lambda_{\mathrm{ex}}=\lambda_{\mathrm{em}}\right)$, respectively [49]. Peak 1 shows the characteristic fluorescence peak of Tyr and Trp residues. Also, peak 2 presents the characteristic fluorescence peak of polypeptide backbone structures, which are associated with the secondary structure. From Figures 5(d) and 5(e), fluorescence intensities of peak 1 and peak 2 reduced significantly after ATRA bound into Pin1, which further indicated that ATRA affected the conformation of Pin1.

\subsection{Computational Studies}

3.3.1. Principal Component Analysis and Free Energy Landscape. Principal component analysis (PCA) is an effective technique to reduce the huge dimension of data set to principal components, showing the main changes of protein-ligand complex and providing some important information [50]. It revealed that the first 15 eigenvectors account for $70.08 \%$ of the total variance, which was equal to the 
previous report of about 70\% [30]. In addition, two important eigenvectors, principal components 1 and 2 (PC 1 and PC 2), contributed about $35.98 \%$ to the total variance with the conformational space.

Based on PCA results, the conformational change of Pin1ATRA complex was explored through free energy landscape (FEL) of PC 1 and PC 2. As shown in Figure 3(a), it is noteworthy that Pin1-ATRA complex has widely two deeper energy basins with energy barriers in the range of $1.0 \mathrm{~kJ} / \mathrm{mol}$ to $4.0 \mathrm{~kJ} / \mathrm{mol}$, indicating that the binding of ATRA to Pin 1 may form two stable conformations. In other words, the process of ATRA binding to Pin1 will cause its dynamics conformational transitions, which corresponds well to the spectroscopic phenomena (Figures 2(a), 2(b), and 5).

According to FEL results, two representative conformational models are displayed in Figures 3(b)-3(g). From Figures 3(b)-3(d), ATRA interacts with residues H59, L61, K63, R69, C113, M130, Q131, F134, and H157. It is observed that the carboxylic acid of ATRA forms a critical hydrogen bond with residue R69 and also forms seven Pi-Alkyl interactions (a type of hydrophobic interaction). In addition, residues K63, Q131, and R69 interact with ATRA via weak van der Waals and salt bridge (a type of electrostatic force). Figures 3(e)-3(g), showing the lowest energy minima, reveal that residues H59, K63, R68, R69, M130, F134, and H157 play an important role in the process of binding ATRA to Pin1 through Pi-Alkyl interaction. Meanwhile, residue K63 forms a salt bridge and residue R69 forms a stable hydrogen bond with ATRA. These observations were similar to previous reports [22], suggesting that the carboxylic acid of ATRA formed salt bridges and hydrogen bonds with residues K63 and R68, and the hydrophobic skeleton of ATRA formed hydrophobic interactions and van der Waals with residues $\mathrm{R} 68, \mathrm{C} 113, \mathrm{M} 130, \mathrm{Q} 131, \mathrm{~F} 134$, and $\mathrm{H} 157$. All in all, the results, which were in line with experimental works, further suggested that the main binding forces were strong hydrophobic interactions, but electrostatic forces, weak van der Waals, and hydrogen bonds cannot be ignored.

3.3.2. MD Simulations. MD simulation is a popular tool to investigate dynamic conformational stability at the atomic level. Root mean square deviation (RMSD) values, an important criterion for assessing conformational stability, of Pin1 and Pin1-ATRA complex were calculated for initial conformations (Figure 4(a)). Two systems are relatively stable with approximately $1.75 \AA$ during 50 ns MD simulations, which suggest that the binding of ATRA to Pin1 is relatively stable. From Figure 4(b), it is noteworthy that RMSD values of ATRA reach a relative equilibrium state after $15 \mathrm{~ns} \mathrm{MD}$ simulations. As seen in Figure 4(c), root mean square fluctuation (RMSF) values, another important indicator for evaluating structural stability, show that two systems share similar fluctuation, except for the region of certain binding residues H59, L61, K63, R68, C113, M130, Q131, F134, and H157.

As shown in Figure 4(d), radius of gyration ( $\mathrm{Rg}$ ) value, a valuable indicator for evaluating the compactness of a protein, of Pin1-ATRA complex was less than that of Pin1 during $50 \mathrm{~ns} \mathrm{MD}$ simulations. The results meant that ATRA entered the active pocket of Pin1, causing its conformation to become loose. From Figure 4(e), hydrogen bonds of Pin1ATRA complex had been present during 50 ns MD simulations, with an average value of about 1 , which was in line with the binding model (Figures 3(d) and 3(g)).

Binding free energy $\left(\Delta G_{\text {bind }}\right)$ of Pin1-ATRA system is predicted to be $-167.19 \mathrm{~kJ} / \mathrm{mol}$, suggesting that the binding process is spontaneous between Pin 1 and ATRA (Table S1). In addition, energy decompositions show that van der Waals energy $\left(\Delta E_{\mathrm{vdw}}\right)$, electrostatic energy $\left(\Delta E_{\text {ele }}\right)$, polar solvation energy $\left(\Delta G_{\text {polar }}\right)$, and nonpolar solvation energy $\left(\Delta G_{\text {nonpolar }}\right)$ are $-120.70,-121.41,88.04$, and $-13.12 \mathrm{~kJ} / \mathrm{mol}$, respectively (Table S1). The result revealed that van der Waals energy, electrostatic energy, and nonpolar solvation were conducive to the binding process of ATRA to Pin1, whereas the polar solvation energy was the opposite. In addition, nonpolar interaction energy $\left(\Delta E_{\mathrm{vdw}}+\Delta G_{\text {nonpolar }}\right)$ and polar interaction energy $\left(\Delta E_{\text {ele }}+\Delta G_{\text {polar }}\right)$ are -133.38 and $-33.37 \mathrm{~kJ} / \mathrm{mol}$, respectively, in Pin1-ATRA system, indicating that both of these energies contribute to the binding. Meanwhile, residue energy contributions also are predicted in Pin1-ATRA system. From Figure 4(f), it is observed that residues K63, R68, and R69 may play a significant role in the binding process between Pin1 and ATRA.

\subsection{Mutant Studies}

3.4.1. Fluorescence Titrations. Fluorescence titrations were utilized to verify the key residues in the binding process of ATRA to Pin1. As shown in Figure 6(a) and Figure S1, ATRA quenched the endogenous fluorescence of Pin1 mutants in different degrees, but K63A and R69A showed the weakest quenching. In addition, the binding constants revealed that mutations of these important residues reduced the binding affinity for ATRA to varying degrees, especially residues K63 and R69 (Figure 6(b) and Figure S2). Previous research illustrated that the carboxylic acid of ATRA formed salt bridges with residues K63 and R68 [22], which was similar to our results, further indicating that these two basic residues were essential for the binding of ATRA to Pin 1 . This observation could provide certain ideas for the subsequent development and design of retinoic acid drugs to inhibit the activity of Pin 1 .

3.4.2. DARTS Assay. DARTS assay was implemented to detect the effect of key residue mutations on the stability of ATRA binding to Pin1. As shown in Figure 6(c), it was observed that mutants $\mathrm{K} 63 \mathrm{~A}$ and R69A were less resistant to proteolysis when bound to ATRA than wild type. This result was consistent with fluorescence titration, which further implied that residues K63 and R69 played an important role in the process of ATRA binding to Pin1.

3.4.3. MD Simulations of Mutants. Using the PyMol plugin Mutagenesis, we predicted that the important residues of Pin 1 would be mutated into alanine mutants. As shown 

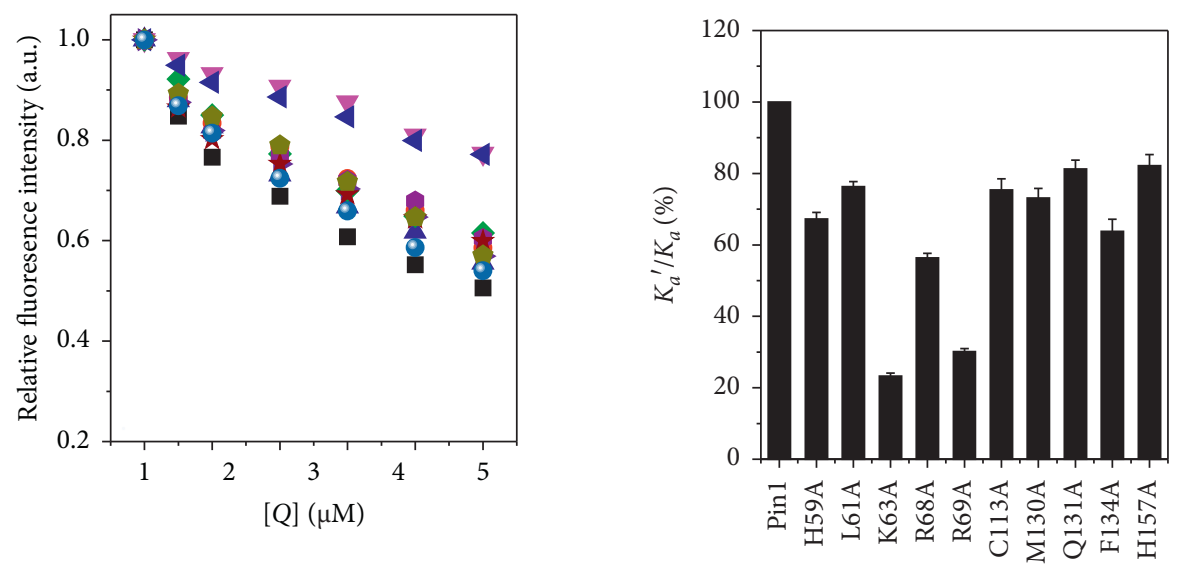
- Pin 1
- L61A
R68A
$>\mathrm{C} 113 \mathrm{~A}$
$\star$ Q131A
- H157A
- $\mathrm{H} 59 \mathrm{~A}$
V K63A
4 R69A
- M130A
- F134A

(a)

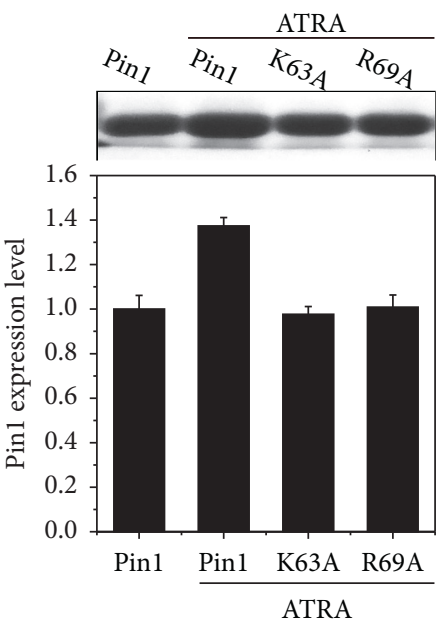

(c)

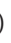

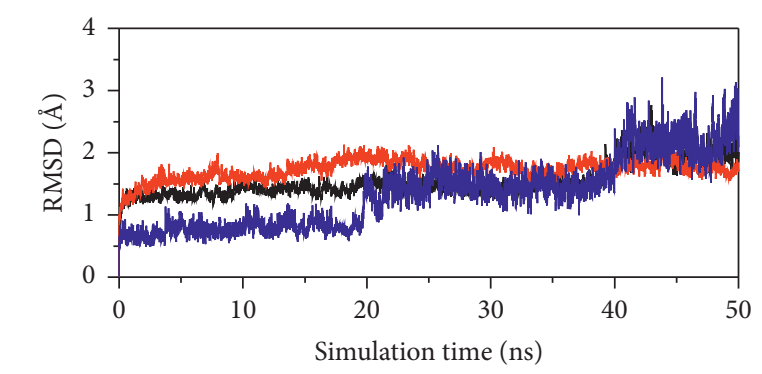

- Pin 1

Pin1-ATRA

_ R69A-ATRA (b)

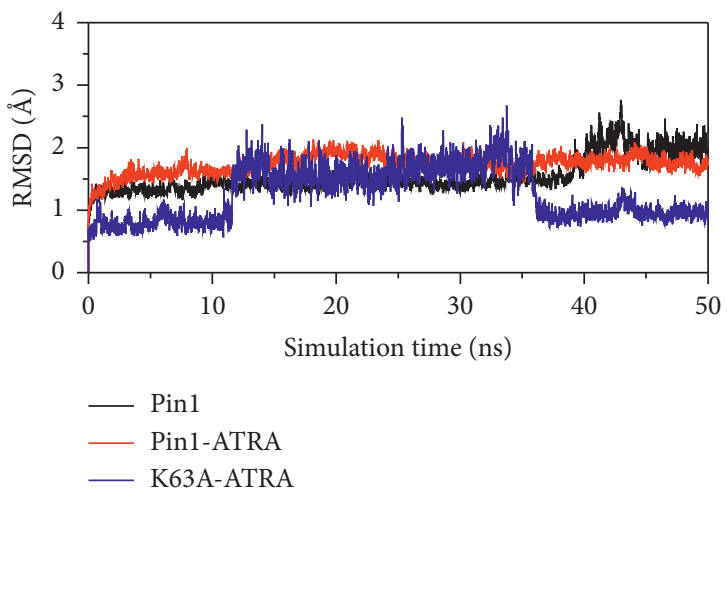

(d)

(e)

Figure 6: ATRA binds to Pin1 through key residues. (a) Fluorescence titration of Pin1 and its mutants in the absence and presence of ATRA; $\lambda_{e x}=295 \mathrm{~nm} ; \mathrm{T}=293 \mathrm{~K} ; \mathrm{c}_{\text {pin } 1 \text { or mutants }}=5 \mu \mathrm{M} ; \mathrm{c}_{\mathrm{ATRA}}=0,0.5,1,2,3,4$, and $5 \mu \mathrm{M}$. (b) Relative binding constant of ATRA to Pin1 and its mutants. (c) DARTS assay of Pin1 and its mutants by ATRA. (d, e) RMSD of K63A-ATRA and R69A-ATRA complex during 50 ns MD simulation. 
in Figure S3, these ten important residues mutations did not destroy the secondary structure of Pin1, which was similar to previous work, indicating that single residue mutations had a limited impact on the overall secondary structure of protein [51]. In addition, the $\mathrm{CD}$ results of mutants K63A, R68A, and R69A were similar to those of wild type, which further confirmed this phenomenon (Figure S4).

Next, we performed MD simulations to explore the effects of residues K63 and R69 mutations on the binding stability of Pin1 and ATRA. During 50 ns MD simulations, the RMSD values of K63A-ATRA and R69A-ATRA systems had been unstable compared with wild type (Figures 6(d) and $6(\mathrm{e}))$. In addition, binding free energies $\left(\Delta G_{\text {bind }}\right)$ of K63A-ATRA and R69A-ATRA systems are -70.81 and $-51.23 \mathrm{~kJ} / \mathrm{mol}$, respectively, which are lower than that of Pin1-ATRA system $(-167.19 \mathrm{~kJ} / \mathrm{mol}$, Table S1). These phenomena indicated that mutations of residues $\mathrm{K} 63 \mathrm{~A}$ and R69A would affect the binding of ATRA to Pin1.

\section{Conclusion}

The present work explains the details of the binding and conformational change of ATRA with Pin1 using fluorescence spectra, circular dichroism, MD simulations, binding free energy, and free energy landscape under physiological conditions. Fluorescence emission spectra showed that the binding mechanism of ATRA to Pin1 was a static quenching process with a moderate binding affinity. Thermodynamic parameters and computational simulations indicated that the binding force was mainly hydrophobic interactions, but other forces were also involved in the binding process of ATRA to Pin1. Circular dichroism, synchronous fluorescence, and three-dimensional fluorescence spectra demonstrated that the binding of ATRA to Pin1 reduces the helical stability of its active center. Free energy landscape and MD simulation displayed that the process of ATRA binding to Pin1 would cause its dynamic conformational transitions. Computational simulations, fluorescence titrations, and DARTS assays demonstrated that residues K63 and R69 played an important role in the binding process between Pin1 and ATRA. In summary, these works help clarify the binding mechanism of ATRA and Pin1 and provide useful information for the application of ATRA as a therapeutic drug in cancer.

\section{Data Availability}

All data included in this study are available upon request to the corresponding author.

\section{Conflicts of Interest}

The authors declare no conflicts of interest.

\section{Authors' Contributions}

GuoFei Zhu, ShaoLi Lyu, and Yang Liu contributed equally to this work.

\section{Acknowledgments}

This work was financially supported by the Science and Technology Foundation of Guizhou Province of China (qian ke he zi [2017]1068) and Guizhou Institute of Technology High-Level Talent Research Startup Project (XJGC20190658).

\section{Supplementary Materials}

Figure S1: fluorescence spectra of Pin1 mutants-ATRA complex. (A-J) Fluorescence spectra of H59A, L61A, K63A, R68A, R69A, C113A, M130A, Q131A, F134A, and H157A in the absence and presence of ATRA, respectively. $\lambda_{e x}=295 \mathrm{~nm}, c_{\text {pin } 1}=5 \mu \mathrm{M}$, and cATRA $=0$ (black line), 0.5 (red line), 1 (blue line), 2 (magenta line), 3 (olive line), 4 (navy line), and 5 (violet line) $\mu \mathrm{M}$. Figure S2: double logarithmic plot of Pin1 mutants-ATRA complex (a) and binding constant (b). Figure S3: schematic diagram of Pin1 alanine mutants. (a, b) Global and local graphs, respectively. Figure S4: CD spectra of Pin1 and its mutants. $c_{\mathrm{Pin} 1}=c_{\mathrm{K} 63 \mathrm{~A}}=c_{\mathrm{R} 68 \mathrm{~A}}=c_{\mathrm{R} 69 \mathrm{~A}}=10 \mu \mathrm{M}$. Table S1: binding free energy and energy decomposition of Pin 1 and its mutants by ATRA using MM/PBSA method. (Supplementary Materials)

\section{References}

[1] Y. Zheng, W. Pu, J. Li et al., "Discovery of a prenylated flavonol derivative as a Pin1 inhibitor to suppress hepatocellular carcinoma by modulating microRNA biogenesis," Chemistry-An Asian Journal, vol. 14, no. 1, pp. 130-134, 2019.

[2] Z. Lu and T. Hunter, "Prolyl isomerase Pin1 in cancer," Cell Research, vol. 24, no. 9, pp. 1033-1049, 2014.

[3] C.-W. Cheng, K.-W. Leong, and E. Tse, "Understanding the role of Pin1 in hepatocellular carcinoma," World Journal of Gastroenterology, vol. 22, no. 45, pp. 9921-9932, 2016.

[4] C.-W. Cheng and E. Tse, "Pin1 in cell cycle control and cancer," Frontiers in Pharmacology, vol. 9, p. 1367, 2018.

[5] L. C. Han, L. H. Yi, L. Y. Cheng et al., "Landscape of Pin1 in the cell cycle," Experimental Biology and Medicine, vol. 240, no. 3, pp. 403-408, 2015.

[6] D. V. Urusova, J.-H. Shim, D. J. Kim et al., "Epigallocatechingallate suppresses tumorigenesis by directly targeting Pin 1 ," Cancer Prevention Research, vol. 4, no. 9, pp. 1366-1377, 2011.

[7] Y. M. Lee and Y.-C. Liou, "Gears-In-Motion: the interplay of WW and PPIase domains in Pin1," Frontiers in Oncology, vol. 8, pp. 469-480, 2018.

[8] A. Zannini, A. Rustighi, E. Campaner, and G. Del Sal, "Oncogenic hijacking of the Pin 1 signaling network," Frontiers in Oncology, vol. 9, p. 94, 2019.

[9] G. Kim, P. Y. Bhattarai, and H. S. Choi, "Peptidyl-prolyl cis/ trans isomerase NIMA-interacting 1 as a molecular target in breast cancer: a therapeutic perspective of gynecological cancer," Archives of Pharmacal Research, vol. 42, no. 2, pp. 128-139, 2019.

[10] H. R. Shin, H. S. Bae, B. S. Kim et al., "Pin1 is a new therapeutic target of craniosynostosis," Human Molecular Genetics, vol. 27, no. 22, pp. 3827-3839, 2018.

[11] Y. Chen, Y.-R. Wu, H.-Y. Yang et al., "Prolyl isomerase Pin1: a promoter of cancer and a target for therapy," Cell Death \& Disease, vol. 9, no. 9, p. 883, 2018. 
[12] D. Yang, W. Luo, J. Wang et al., "A novel controlled release formulation of the Pin1 inhibitor ATRA to improve liver cancer therapy by simultaneously blocking multiple cancer pathways," Journal of Controlled Release, vol. 269, pp. 405422, 2018.

[13] M. V. Giuli, P. N. Hanieh, E. Giuliani et al., "Current trends in ATRA delivery for cancer therapy," Pharmaceutics, vol. 12, no. 8, p. 707, 2020.

[14] X. H. Liao, A. L. Zhang, M. Zheng et al., "Chemical or genetic Pin1 inhibition exerts potent anticancer activity against hepatocellular carcinoma by blocking multiple cancer-driving pathways," Scientific Reports, vol. 7, no. 1, pp. 43639-43711, 2017.

[15] S. Huang, Y. Chen, Z.-M. Liang et al., "Targeting Pin1 by alltrans retinoic acid (ATRA) overcomes tamoxifen resistance in breast cancer via multifactorial mechanisms," Frontiers in Cell and Developmental Biology, vol. 7, p. 322, 2019.

[16] Z. Zhang, W. Yu, M. Zheng et al., "Pin1 inhibition potently suppresses gastric cancer growth and blocks PI3K/AKT and Wnt/ $\beta$-catenin oncogenic pathways," Molecular Carcinogenesis, vol. 58, no. 8, pp. 1450-1464, 2019.

[17] W.-N. Shi, S.-X. Cui, Z.-Y. Song et al., "Overexpression of SphK2 contributes to ATRA resistance in colon cancer through rapid degradation of cytoplasmic RXR $\alpha$ by K48/K63linked polyubiquitination," Oncotarget, vol. 8, no. 24, pp. 39605-39617, 2017.

[18] Z. Liu, G. Ren, C. Shangguan et al., "ATRA inhibits the proliferation of DU145 prostate cancer cells through reducing the methylation level of HOXB13 gene," PLoS One, vol. 7, no. 7, p. e40943, 2012.

[19] S. Bagheri, "The effect of arsenic trioxide on all-trans retinoic acid binding to human serum albumin," Journal of Fluorescence, vol. 29, no. 6, pp. 1277-1283, 2019.

[20] X. Lian, Y. M. Lin, S. Kozono et al., "Pin1 inhibition exerts potent activity against acute myeloid leukemia through blocking multiple cancer-driving pathways," Journal of Hematology \& Oncology, vol. 11, no. 1, pp. 73-14, 2018.

[21] S. Kozono, Y. M. Lin, H. S. Seo et al., "Arsenic targets Pin1 and cooperates with retinoic acid to inhibit cancer-driving pathways and tumor-initiating cells," Nature Communications, vol. 9, no. 1, pp. 3069-3117, 2018.

[22] S. Wei, S. Kozono, L. Kats et al., "Active Pin1 is a key target of alltrans retinoic acid in acute promyelocytic leukemia and breast cancer," Nature Medicine, vol. 21, no. 5, pp. 457-466, 2015.

[23] J.-Z. Wang, T. Lin, T. Teng, S.-S. Xie, G.-F. Zhu, and L.-F. Du, "Spectroscopic studies on the irreversible heat-induced structural transition of Pin1," Spectrochimica Acta Part A: Molecular and Biomolecular Spectroscopy, vol. 78, no. 1, pp. 142-147, 2011.

[24] J.-Z. Wang, J. Liu, T. Lin et al., "Aluminum(III) interferes with the structure and the activity of the peptidyl-prolyl cis-trans isomerase (Pin1): a new mechanism contributing to the pathogenesis of Alzheimer's disease and cancers?" Journal of Inorganic Biochemistry, vol. 126, pp. 111-117, 2013.

[25] W. L. DeLano, "Pymol: an open-source molecular graphics tool," CCP4 Newsletter on protein crystallography, vol. 40, no. 1, pp. 82-92, 2002.

[26] T. Schwede, J. Kopp, N. Guex, and M. C. Peitsch, "SwissModel: an automated protein homology-modeling server," Nucleic Acids Research, vol. 31, no. 13, pp. 3381-3385, 2003.

[27] S. Pronk, S. Páll, R. Schulz et al., "Gromacs 4.5: a highthroughput and highly parallel open source molecular simulation toolkit," Bioinformatics, vol. 29, no. 7, pp. 845-854, 2013.
[28] K. Lu, X. Wang, Y. Chen et al., "Identification of two potential glycogen synthase kinase $3 \beta$ inhibitors for the treatment of osteosarcoma," Acta Biochimica et Biophysica Sinica, vol. 50, no. 5, pp. 456-464, 2018.

[29] R. Kumari, R. Kumar, and A. Lynn, "g_mmpbsa-A GROMACS tool for high-throughput MM-PBSA calculations," Journal of Chemical Information and Modeling, vol. 54, no. 7, pp. 1951-1962, 2014.

[30] E. Papaleo, P. Mereghetti, P. Fantucci, R. Grandori, and L. De Gioia, "Free-energy landscape, principal component analysis, and structural clustering to identify representative conformations from molecular dynamics simulations: the myoglobin case," Journal of Molecular Graphics and Modelling, vol. 27, no. 8, pp. 889-899, 2009.

[31] P. S. Srikumar, K. Rohini, and P. K. Rajesh, "Molecular dynamics simulations and principal component analysis on human laforin mutation W32G and W32G/K87A," The Protein Journal, vol. 33, no. 3, pp. 289-295, 2014.

[32] A. Singh, P. Somvanshi, and A. Grover, "Drug repurposing against arabinosyl transferase (EmbC) of mycobacterium tuberculosis: essential dynamics and free energy minima based binding mechanics analysis," Gene, vol. 693, pp. 114126, 2019.

[33] P. Sang, X. Du, L.-Q. Yang, Z.-H. Meng, and S.-Q. Liu, "Molecular motions and free-energy landscape of serine proteinase $\mathrm{K}$ in relation to its cold-adaptation: a comparative molecular dynamics simulation study and the underlying mechanisms," RSC Advances, vol. 7, no. 46, pp. 28580-28590, 2017.

[34] A. Singh, P. Somvanshi, and A. Grover, "Pyrazinamide drug resistance in RpsA mutant $(\triangle 438 \mathrm{~A})$ of Mycobacterium tuberculosis: dynamics of essential motions and free-energy landscape analysis," Journal of Cellular Biochemistry, vol. 120, no. 5, pp. 7386-7402, 2019.

[35] B. Lomenick, R. Hao, N. Jonai et al., "Target identification using drug affinity responsive target stability (DARTS)," Proceedings of the National Academy of Sciences, vol. 106, no. 51, pp. 21984-21989, 2009.

[36] J.-Z. Wang, L. Xi, G.-F. Zhu et al., "The acidic pH-induced structural changes in Pin1 as revealed by spectral methodologies," Spectrochimica Acta Part A: Molecular and Biomolecular Spectroscopy, vol. 98, pp. 199-206, 2012.

[37] J. R. Lakowicz, Principles of Fluorescence Spectroscopy, Springer Science \& Business Media, Berlin, Germany, 2013.

[38] J. Wang, T. Lin, G. Zhu, and L. Du, "Stability of Pin1 as revealed by thermal and spectroscopic studies," Journal of Molecular Structure, vol. 975, no. 1-3, pp. 310-316, 2010.

[39] J. Yu, X. Li, H. Liu, Y. Peng, X. Wang, and Y. Xu, "Interaction behavior between five flavonoids and pepsin: spectroscopic analysis and molecular docking," Journal of Molecular Structure, vol. 1223, p. 128978, 2020.

[40] A. S. Abdelhameed, "Insight into the interaction between the HIV-1 integrase inhibitor elvitegravir and bovine serum albumin: a spectroscopic study," Journal of Spectroscopy, vol. 2015, Article ID 435674, 9 pages, 2015.

[41] S. Lyu and W. Wang, "Spectroscopic methodologies and computational simulation studies on the characterization of the interaction between human serum albumin and astragalin," Journal of Biomolecular Structure and Dynamics, vol. 39, pp. 1-12, 2020.

[42] G.-F. Zhu, Y. Wang, L. Xi, J. Liu, H. Wang, and L.-F. Du, "Spectroscopy and molecular docking studies on the binding of propyl gallate to human serum albumin," Journal of Luminescence, vol. 159, pp. 188-196, 2015. 
[43] M. Chen, X. Zuo, Q. Xu, R. Wang, S. Fan, and H. Wu, "Investigating the interaction of nanodiamonds with human serum albumin and induced cytotoxicity," Journal of Spectroscopy, vol. 2019, Article ID 4503137, 9 pages, 2019.

[44] A. A. Al-Mehizia, A. H. Bakheit, S. Zargar, M. A. Bhat, M. M. Asmari, and T. A. Wani, "Evaluation of biophysical interaction between newly synthesized pyrazoline pyridazine derivative and bovine serum albumin by spectroscopic and molecular docking studies," Journal of Spectroscopy, vol. 2019, Article ID 3848670, 12 pages, 2019.

[45] W. Du, T. Teng, C.-C. Zhou, L. Xi, and J.-Z. Wang, "Spectroscopic studies on the interaction of bovine serum albumin with ginkgolic acid: binding characteristics and structural analysis," Journal of Luminescence, vol. 132, no. 5, pp. 1207-1214, 2012.

[46] P. D. Ross and S. Subramanian, "Thermodynamics of protein association reactions: forces contributing to stability," Biochemistry, vol. 20, no. 11, pp. 3096-3102, 1981.

[47] W. Cao, Y. Fang, T. Wu et al., "Insights from multispectral and molecular docking investigation on the xanthine oxidase inhibition by 1,4-dicaffeoylquinic acid," Journal of Molecular Structure, vol. 1219, p. 128475, 2020.

[48] L. Xi, Y. Wang, Q. He, Q. Zhang, and L. Du, "Interaction between Pin1 and its natural product inhibitor epigallocatechin-3-gallate by spectroscopy and molecular dynamics simulations," Spectrochimica Acta Part A: Molecular and Biomolecular Spectroscopy, vol. 169, pp. 134-143, 2016.

[49] M. Raza, Y. Jiang, Y. Wei, A. Ahmad, A. Khan, and Y. Qipeng, "Insights from spectroscopic and in-silico techniques for the exploitation of biomolecular interactions between Human serum albumin and Paromomycin," Colloids and Surfaces B: Biointerfaces, vol. 157, pp. 242-253, 2017.

[50] J. Chen, "Functional roles of magnesium binding to extracellular signal-regulated kinase 2 explored by molecular dynamics simulations and principal component analysis," Journal of Biomolecular Structure and Dynamics, vol. 36, no. 2, pp. 351-361, 2018.

[51] J.-Z. Wang, L. Xi, T. Lin, Y. Wang, G.-F. Zhu, and L.-F. Du, "The structural and functional role of the three tryptophan residues in Pin1," Journal of Photochemistry and Photobiology B: Biology, vol. 146, pp. 58-67, 2015. 